\title{
(2) OPEN ACCESS \\ Mortality and pulmonary complications in patients undergoing upper extremity surgery at the peak of the SARS-CoV-2 pandemic in the UK: a national cohort study
}

\author{
Benjamin John Floyd Dean (1) , The Corona Hands Collaborative
}

Orthopaedic Surgery, Department of Orthopaedics Rheumatology and Musculoskeletal Sciences, University of Oxford Nuffield, Oxford, UK

\section{Correspondence to} Benjamin John Floyd Dean, Orthopaedic Surgery, Department of Orthopaedics Rheumatology and Musculoskeletal Sciences, University of Oxford Nuffield, Oxford OX3 7LD, UK: bendean1979@gmail.com

Received 8 August 2020 Revised 15 September 2020 Accepted 21 September 2020 Published Online First 9 October 2020

\section{Sinked}

- http://dx.doi.org/10.1136/ bmjqs-2020-012544

\section{Check for updates}

(c) Author(s) (or their employer(s)) 2021. Re-use permitted under CC BY-NC. No commercial re-use. See rights and permissions. Published by BMJ.

\section{To cite: Dean BJF.} BMJ Qual Saf 2021;30:283-291.

\begin{abstract}
Introduction This study reports the 30-day mortality, SARS-CoV-2 complication rate and SARS-CoV-2-related hospital processes at the peak of the first wave of the pandemic in the UK.

Methods This national, multicentre, cohort study at 74 centres in the UK included all patients undergoing any surgery below the elbow at the peak of the UK pandemic. The primary outcome measure was 30-day postoperative mortality and was assessed in all enrolled patients. The secondary outcomes were SARS-CoV-2 complication rates and overall complication rates. A clinician survey relating to SARS-CoV-2 safety processes was carried out for each participating centre.

Results This analysis includes 1093 patients who underwent upper limb surgery from the 1 to 14 April 2020 inclusively. The overall 30-day mortality was $0.09 \%$ (1 pre-existing SARS-CoV-2 pneumonia) and the mortality of day case surgery was zero. Most centres (96\%) screened patients for symptoms prior to admission, only $22 \%$ routinely tested for SARS-CoV-2 prior to admission. The SARS-CoV-2 complication rate was $0.18 \%$ ( 2 pneumonias) and the overall complication rate was $6.6 \%$ (72 patients). Both SARS-CoV-2-related complications occurred in patients who had been hospitalised for a prolonged period before their surgery and a total of 19 patients (1.7\%) were SARS-CoV-2 positive.

Conclusions The SARS-CoV-2-related complication rate for upper limb surgery even at the peak of the UK pandemic was low at $0.18 \%$ and the mortality was zero for patients admitted on the day of surgery. Urgent surgery should not be delayed pending the results of SARS-CoV-2 testing. Routine SARS-CoV-2 testing for day case upper limb surgery not requiring general anaesthesia may be excessive and have unintended negative impacts.
\end{abstract}

\section{INTRODUCTION}

The SARS-CoV-2 pandemic has had significant implications for the delivery of both urgent and planned surgical services around the world. In April in the $\mathrm{UK}$, the daily number of lab-confirmed cases peaked, and daily number of deaths reached a plateaued peak. ${ }^{1}$ At this time point planned and elective surgery was largely stopped, while urgent and emergency surgery continued, in the context of more limited access to theatres and with modified perioperative practices.

A recent global cohort study demonstrated that postoperative pulmonary complications occurred in half of patients with perioperative SARS-CoV-2 infection and were associated with high mortality. ${ }^{2}$ However, there are significant potential issues regarding the meaning and generalisability of this study; there are methodological concerns as regard the way in which patients were selected for inclusion, and it is arguable that they cannot be generalised to surgery of the upper extremity and more minor surgery under local anaesthetic, given the case mix.

Recently, planned care has started to resume in the UK, with guidance from NHS England and the British Orthopaedic Association both emphasising the need to separate the elective pathways from the emergency pathways. ${ }^{34}$ This guidance has advised SARS-CoV-2 testing for all emergency and elective admissions (including day surgery), and 14 days of self-isolation for the family units of any patient undergoing elective surgery. This guidance is proposed despite a substantial drop in both the daily number of lab-confirmed cases and daily deaths. More recently, the National Institute for Health and Care Excellence has published recent guidance relating to planned care which has advised comprehensive social distancing and hand hygiene measures for 14 days before any admission, a SARS-CoV-2 test 
3 days before admission and self-isolation from the day of the test until admission. ${ }^{5}$ Questions remain regarding the ongoing delivery of both urgent and planned surgery in the context of the current global SARS-CoV-2 surges.

\section{Aims and objectives}

Given that a significant volume of surgery continued during the pandemic peak in the UK, our aim was to assess the safety of upper extremity surgery below the elbow that was carried out at this time when it was not feasible or advisable to isolate family units for 14 days before surgery or routinely test for SARS-CoV-2 before surgery. Specifically, our primary objective was to assess the 30-day mortality of patients undergoing upper extremity surgery below the elbow at the peak of the pandemic. Our secondary objectives were to assess the SARS-CoV-2-related complication rate, the non-SARS-CoV-2 complication rates, the re-operation rate and the nature of local SARS-CoV-2 hospital safety processes.

\section{METHODS}

We carried out a national multicentre observational cohort study in patients undergoing any form of upper extremity surgery below the elbow with or without SARS-CoV-2 infection at 74 institutions in the UK. We collected only routine, anonymised data with no change to clinical care pathways. The study was registered at each site as a service evaluation project; and at the lead centre (Nuffield Orthopaedic Centre), it was approved by the clinical audit team, with registration number 6091.

\section{Participants}

Each participating institution included all patients undergoing any form of upper extremity surgery below the elbow from 1 April to 14 April inclusive, this included the Easter bank holiday weekend. Surgery was defined as any procedure done in any form of operating theatre or procedure room under general, regional or local anaesthesia. Patients undergoing surgery for any indication were eligible, including benign disease, cancer, trauma, infection and nerve compression. The study included children and adults. Patients having surgery on other body parts in addition to the hand/wrist procedure, whether this was at the same sitting or another sitting during the same hospital episode, were excluded.

Participating hospitals used both electronic and paper records in order to identify all patients fulfilling the eligibility criteria. Several units had gathered a patient list prospectively, while others gathered data retrospectively. Site investigators were provided with a range of written materials including a protocol which detailed the specifics of eligibility and data input. Due to the lack of widespread SARS-CoV-2 testing at this time point, there was a concern that including only those who tested positive would introduce a significant degree of bias into the study, and it was acknowledged that it would be likely that a number of patients included within the study would be asymptomatic positives, but there would be no way of defining the exact size of this subgroup.

The clinician survey was completed remotely in electronic format by the lead consultant surgeon for each institution. This survey was piloted locally and if any questions were unclear then these were addressed by email or telephone communication. Level 2 personal protective equipment (PPE) was defined as at least an FFP3 respirator mask, eye protection and surgical gown. Level 1 was a surgical gown with a fluid-resistant surgical mask.

\section{Patient data}

Data were collected from the patient record using a standardised Excel spreadsheet and securely returned by the lead author via institutional email or secure file transfer. Demographic variables recorded included age, sex, specific comorbidities, smoking status. The SARS-CoV-2 status was recorded as positive (positive swab PCR presurgery, positive swab PCR postsurgery, CT chest positive presurgery, CT chest positive postsurgery, chest X-ray (CXR) or clinical suspicion presurgery, CXR or clinical suspicion postsurgery) or negative (no suspicion, no suspicion and swab PCR negative presurgery). Clinical diagnosis consistent with SARS-CoV-2 infection was made by a senior physician and based on clinical presentation of symptoms highly indicative of SARS-CoV-2 infection, including cough, fever and myalgia.

Operative variables included indication for surgery (trauma, infection, cancer, nerve compression, other), procedure completed and anaesthesia used (local with epinephrine, local without epinephrine, local with sedation, regional, regional and local, regional with sedation \pm local, general only, general and local, general and regional and other). Emergency surgery was defined as procedures classified by the National Confidential Enquiry into Patient Outcome and Death as immediate, urgent or expedited. The senior local principal investigator for each hospital was asked to confirm data completeness and that all eligible patients had been entered into the database. The length of stay, and nature of surgical follow-up were also recorded.

\section{Outcomes}

The primary outcome was 30-day mortality, with the day of surgery defined as day 0 . The other secondary outcome measures were the rate of SARS-CoV-2 complications, the rate of non-SARS-CoV-2 complications and re-operation rate. The specific complications recorded were COVID-19 pneumonia, acute respiratory distress syndrome (ARDS), intensive care unit (ICU) admission, sepsis, renal failure, superficial infection, deep infection, tendon rupture, nerve injury, 
fracture displacement, chronic regional pain syndrome and other. A SARS-CoV-2 complication was defined as any patient with any form of positive COVID-19 status and any form of COVID-19-related complication (COVID-19 pneumonia, ARDS, ICU admission, renal failure, sepsis).

\section{Statistical analysis}

The study was done according to STROBE guidelines for observational studies. ${ }^{6}$ Summary statistics (mean, $\mathrm{SD}$, median and IQR) were calculated for continuous variables and number of observation and percentages used for categorical data. Patient data were summarised by the occurrence of an outcome event, if sufficient events were considered to have taken place.

Logistic regression was used to assess the association of key variables of interest with occurrence of a complication with 30 days of surgery, and ORs and 95\% CIs calculated. Due to the relatively low event rates, modelling of any complication (not mortality or re-operation) were carried out. Additionally, the number of variables was limited with an overall comorbidity variable (any one or more of hypertension, ischaemic heart disease, diabetes, stroke/transient ischaemic attack, chronic obstructive pulmonary disease or chronic kidney disease) prior to any modelling. Factors included in the model were age, gender, comorbidity (one or more vs none), smoking, surgical indications (infection or non-infection), urgency of surgery (urgent/immediate or not), regional/general anaesthetic or not and tourniquet use. A sensitivity analysis adjusted for potential clustering by centre using generalised estimating equation (GEE) model with logit function and assuming exchangeable correlation structure with Huber-White Sandwich variance estimator. Analyses were done using Stata V.15.1.

\section{RESULTS}

\section{Centres}

In total 74 UK centres participated (see table 1 for characteristics), of which 17 (23\%) were designated major trauma centres and 28 (38\%) had a dedicated hand on-call service. The vast majority (72 centres, 97\%) stopped all elective surgery, with most stopping elective surgery by $22 \mathrm{March}$. The median catchment population was $500 \mathrm{k}$ (IQR $350 \mathrm{k}-780 \mathrm{k}$ ). At only 24 surgical centres $(32 \%)$ did surgeons assess patients clinically for the first time on the day of surgery.

\section{Surgical capacity}

Most centres had access to a dedicated daily theatre list with general anaesthesia availability (58 centres, $78 \%)$, while around half had daily access to regional anaesthesia (36 centres, 49\%). Details on the facilities used by centres are provided in table 2. Main theatres were used by 70 centres (95\%) during this time period, and in 6 centres (8\%) this was new capacity in main theatres as a result of the pandemic. A minor

\begin{tabular}{|c|c|}
\hline Variable & $\mathrm{n}(\%)^{*}$ \\
\hline Major trauma centre & $17(23)$ \\
\hline Population size & $709(753) ; 500(350,780)$ \\
\hline Dedicated 24-hour on-call specialist service & $28(38)$ \\
\hline \multicolumn{2}{|c|}{ Were patients assessed for the first time in person on the day of surgery? } \\
\hline No & $45(61)$ \\
\hline Yes & $24(32)$ \\
\hline Unsure & $5(7)$ \\
\hline \multicolumn{2}{|l|}{ Date stopping of elective practice } \\
\hline Stopped & $72(97)$ \\
\hline 8-14 March & $7(10)$ \\
\hline 15-21 March & $37(51)$ \\
\hline 22-28 March & $22(31)$ \\
\hline 29 March-1 April & $6(8)$ \\
\hline Daily general anaesthesia access & $58(78)$ \\
\hline \multicolumn{2}{|l|}{ Daily regional anaesthesia access } \\
\hline No & $36(49)$ \\
\hline Yes & $36(49)$ \\
\hline Unsure & $2(3)$ \\
\hline
\end{tabular}

$(\%), n=74$.

ops theatre was used by 20 centres (26\%) and in 7 centres (9\%) this was new capacity. A clinic procedure room was used by 31 centres (42\%), and in 15 centres $(20 \%)$ this was new capacity. An emergency department procedure room was used by 23 centres (31\%), and in 8 centres $(11 \%)$ this was new capacity. Theatre in a private hospital was used by 19 centres $(26 \%)$ and in 16 centres (22\%) this was new capacity.

\section{SARS-CoV-2 safety processes}

Additional centre SARS-CoV-2-related precautions are summarised in table 3 . Screening questions were used by 71 centres $(96 \%)$ and questions about contacts by 37 centres (50\%). SARS-CoV-2 swab PCR was not undertaken for any patients in 54 centres (73\%). In 18 centres (24\%), SARS-CoV-2 swabbing was performed routinely, in two centres (3\%) swabbing began during the study period, in one centre (1\%) it was performed for inpatient cases and not day case surgery, and in one centre $(1 \%)$ it was performed for general anaesthetic (GA) cases only. Chest imaging (X-ray or CT) was conducted in just three centres (4\%). A dedicated day surgery unit was available for all day surgery patients in 35 centres (47\%) and for some day surgery patients in 16 centres $(22 \%)$. In centres in which patients were first assessed face to face by the surgeon on the day of surgery, the median proportion of patient cancelled on the day was 10\% (IQR 5\%-30\%).

Patients were kept fully isolated from known SARSCoV-2-positive patients in 72 centres (97\%) and partially in 2 centres (3\%). For soft tissue surgery, 23 centres (31\%) used level 1 PPE, 36 centres (49\%) used level 2 and 15 centres used a mixture (20\%). For 
Table 2 Facilities used for surgery

\begin{tabular}{lc}
\hline (n (\%)) & \\
\hline Main theatre & $4(5)$ \\
No & $6(8)$ \\
Yes and not used pre-COVID & $64(87)$ \\
Yes and used pre-COVID & \\
Minor ops theatre & $54(73)$ \\
No & $7(9)$ \\
Yes and not used pre-COVID & $13(18)$ \\
Yes and used pre-COVID & \\
Clinic procedure room & $43(58)$ \\
\hline No & $15(20)$ \\
Yes and not used pre-COVID & $16(22)$ \\
Yes and used pre-COVID & \\
Procedure room in Emergency Department & $51(69)$ \\
No & $8(11)$ \\
Yes and not used pre-COVID & $15(20)$ \\
Yes and used pre-COVID & \\
Theatre in private hospital & $55(74)$ \\
\hline No & $16(22)$ \\
Yes and not used pre-COVID & $3(4)$ \\
\hline Yes and used pre-COVID & \\
\hline
\end{tabular}

surgery which involved drilling into bone 64 centres $(86 \%)$ used level 2 PPE, 6 centres (8\%) used a mixture and 4 centres $(5 \%)$ used level 1 . Hospital policy was for patients to wear a face mask at all times in 13 centres (18\%), just for the surgery in 19 centres (26\%), no mask wear policy in 37 centres (50; respondents were unsure in 5 centres (7\%)).

When asked regarding the surgical procedures concerning an urgent case of a patient with suspected or proven SARS-CoV-2 infection requiring urgent surgery, all centres $(100 \%)$ stated that this involved the use of full level 2 PPE and patient isolation. In 41 centres (55\%), procedures did not involve a specific designated theatre or ward, in 14 centres (19\%) procedures involved a specific designated theatre without a specific designated ward, in 11 centres $(15 \%)$ procedures involved a specific designated theatre and ward, while in the remaining 8 centres $(11 \%)$ procedures involved a specific designated ward without a specific designated theatre.

\section{PATIENTS}

The patient demographics are shown in table 4. A total of 1093 patients underwent upper limb surgery from 1 to 14 April 2020 inclusively at the 74 centres. There were 698 males and 395 females, mean age 42 (SD 21) years. A total of 152 patients (13.9\%) were under 18 years of age.

\section{SURGERY}

The details relating to surgery are shown in table 5 . The most common indications for surgery were trauma (88\%) and infection (8\%). Overall, 915 (84\%)
Table 3 Additional COVID-related precautions

\begin{tabular}{|c|c|}
\hline Variable & $\mathrm{n}(\%)^{*}$ \\
\hline \multicolumn{2}{|c|}{ Pre-admission symptoms screening questions } \\
\hline No & $2(3)$ \\
\hline Yes & $71(96)$ \\
\hline Unsure & $1(1)$ \\
\hline \multicolumn{2}{|l|}{ Potential contacts questions } \\
\hline No & $18(24)$ \\
\hline Yes & $37(50)$ \\
\hline Unsure & $19(26)$ \\
\hline \multicolumn{2}{|l|}{ COVID-19 swab } \\
\hline No & $54(73)$ \\
\hline Yes & $18(24)$ \\
\hline Yes_only certain patients & $2(3)$ \\
\hline Pre-op chest $\mathrm{X}$-ray or $\mathrm{CT}$ & $3(4)$ \\
\hline Proportion of cancellation $(n=39)$ & $25(29) ; 10(5,30)$ \\
\hline \multicolumn{2}{|c|}{ Patients isolated from known COVID-19-positive patients } \\
\hline Yes-all & $72(97)$ \\
\hline \multicolumn{2}{|l|}{ Mask policy } \\
\hline Yes—at all times & $13(18)$ \\
\hline Yes-surgery only & $19(26)$ \\
\hline No & $37(50)$ \\
\hline Unsure & $5(7)$ \\
\hline \multicolumn{2}{|l|}{ PPE level bone } \\
\hline 1 & $4(5)$ \\
\hline Mixture of 1 and 2 & $6(8)$ \\
\hline 2 or above & $64(86)$ \\
\hline \multicolumn{2}{|l|}{ PPE level soft tissue } \\
\hline 1 & $23(31)$ \\
\hline Mixture of 1 and 2 & $15(20)$ \\
\hline 2 or above & $36(49)$ \\
\hline \multicolumn{2}{|c|}{ Procedure for urgent SARS-CoV-2-positive cases } \\
\hline PPE/lsolation only & $41(55)$ \\
\hline PPE/Isolation+theatre+ward & $11(15)$ \\
\hline PPE/lsolation+ward only & $8(11)$ \\
\hline PPE/Isolation+theatre only & $14(19)$ \\
\hline
\end{tabular}

Mean (SD), median (IQR) is given for continuous variables instead of $n$ $(\%), n=74$.

PPE, personal protective equipment.

procedures were undertaken as a day case; 330 procedures were done under GA (30\%), of which 206 (62\%) were as a day-case, 188 under regional block (17\%), of which 157 (84\%) were discharged on the same day and 575 under local anaesthetic only (53\%), and of these $363(70 \%)$ were a day-case.

\section{Mortality and complications including SARS-CoV-2 status}

The complications and factors associated with complications are shown in tables 6 and 7. The overall 30-day mortality was $0.09 \%$ (one SARS-CoV-2 pneumonia) and the mortality of day-case surgery was zero. The only death reported was in a patient over 60 years of age who had been already hospitalised with a suspected SARS-CoV-2 pneumonia who later during this medical 
Table 4 Patient demographics

Patient demographics n (\%)*

\begin{tabular}{|c|c|c|c|}
\hline \multirow[b]{2}{*}{ Variable } & \multicolumn{2}{|c|}{ Postsurgery complications } & \multirow[b]{2}{*}{ Total } \\
\hline & No $(n=1021)$ & Yes $(n=72)$ & \\
\hline \multicolumn{4}{|l|}{ Sex } \\
\hline Male & $651(64)$ & $47(65)$ & $698(64)$ \\
\hline Female & $370(36)$ & $25(35)$ & $395(36)$ \\
\hline \multirow[t]{2}{*}{ Age (years) } & $41(21) ;$ & $50(20) ;$ & $42(21) ;$ \\
\hline & 40 (26 to 57$)$ & 49 (38 to 64$)$ & 41 (27 to 58) \\
\hline \multicolumn{4}{|l|}{ Comorbidities } \\
\hline Asthma & $61(6)$ & $5(7)$ & $66(6)$ \\
\hline Hypertension & $115(11)$ & $12(17)$ & $127(12)$ \\
\hline Ischaemic heart disease & $25(2)$ & $7(10)$ & $32(3)$ \\
\hline Diabetes & $42(4)$ & $7(10)$ & $49(4)$ \\
\hline Smoking & $137(13)$ & $14(19)$ & $151(14)$ \\
\hline Stroke/TIA & $16(2)$ & $2(3)$ & $18(2)$ \\
\hline COPD & $18(2)$ & $2(3)$ & $20(2)$ \\
\hline Chronic kidney disease & $13(1)$ & $1(1)$ & $14(1)$ \\
\hline \multicolumn{4}{|l|}{ COVID-19 status } \\
\hline No suspicion & $997(98)$ & $65(90)$ & $1062(97)$ \\
\hline Swab negative & $9(1)$ & $3(4)$ & $12(1)$ \\
\hline CXR/Clinical presurgery & $12(1)$ & $3(4)$ & $15(1)$ \\
\hline $\begin{array}{l}\text { Swab/CT positive } \\
\text { (presurgery/postsurgery) }\end{array}$ & $3(0)$ & $1(<1)$ & $4(<1)$ \\
\hline
\end{tabular}

admission developed a bacterial hand infection which required surgical treatment; this patient's SARS-CoV-2 treatment was subsequently not escalated beyond the ward level due to medical comorbidity.

The SARS-CoV-2 complication rate was $0.18 \%$ (2 pneumonias, including the mortality above, both confirmed by swab or CT) and the overall complication risk of $6.6 \%$ (72 patients). Both SARS-CoV-2related complications occurred in patients who had been hospitalised for a prolonged period before their upper extremity surgery as part of this study. The non-fatal SARS-CoV-2 pneumonia was in a patient who had multiple debridements for a degloving injury of the upper limb as an inpatient and developed the SARS-CoV-2 pneumonia clinically after the third operation. A total of 19 patients (1.7\%) were SARS-CoV-2 positive of which 17 were confirmed before surgery ( 2 on swab, 15 on CXR/clinical suspicion) and 2 after surgery ( 1 on swab and 1 on CT).

The most common complications were bacterial surgical site infection in 33 cases (3\%), tendon rupture in 9 cases $(0.8 \%)$ and fracture displacement in 7 cases $(0.6 \%)$. Most centres $(71.96 \%)$ were only screening patients for symptoms prior to admission and only $20(24 \%)$ were carrying out SARS-CoV-2 swabbing. Findings from the logistic regression of factors with occurrence of any complications in table 7 . There was an association of greater age, surgical indication (infection) and regional or GA use with increased likelihood of a complication when analysed concurrently (results

Table 5 Surgery-related characteristics

Surgery-related characteristics $\mathrm{n}(\%) 5$

\begin{tabular}{|c|c|c|c|}
\hline \multirow[b]{2}{*}{ Variable } & \multicolumn{2}{|c|}{ Postsurgery complications } & \multirow[b]{2}{*}{ Total } \\
\hline & No $(n=1021)$ & Yes $(n=72)$ & \\
\hline \multicolumn{4}{|l|}{ Urgency of surgery } \\
\hline Urgent & $345(34)$ & $31(43)$ & $376(34)$ \\
\hline Expedited & $645(63)$ & $39(54)$ & $684(63)$ \\
\hline Immediate & $15(1)$ & $2(3)$ & $17(2)$ \\
\hline Elective & $16(2)$ & $0(0)$ & $16(2)$ \\
\hline \multicolumn{4}{|l|}{ Surgery indications } \\
\hline Trauma & $910(89)$ & $52(72)$ & $962(88)$ \\
\hline Infection & $74(7)$ & $17(24)$ & $91(8)$ \\
\hline Cancer & $13(1)$ & $0(0)$ & $13(1)$ \\
\hline Nerve compression & $2(0)$ & $1(1)$ & $3(<1)$ \\
\hline Other & $22(2)$ & $2(3)$ & $24(2)$ \\
\hline \multicolumn{4}{|l|}{ Anaesthetic } \\
\hline Local without epinephrine & $363(36)$ & $13(18)$ & $376(34)$ \\
\hline Local with epinephrine & $188(18)$ & $11(15)$ & $199(18)$ \\
\hline General only & $144(14)$ & $17(24)$ & $161(15)$ \\
\hline General and local & $130(13)$ & $12(17)$ & $142(13)$ \\
\hline General and regional & $24(2)$ & $3(4)$ & $27(2)$ \\
\hline Regional only & $115(11)$ & $13(18)$ & $128(12)$ \\
\hline Regional and local & $15(2)$ & $1(1)$ & $16(2)$ \\
\hline Regional and sedation $+/-$ & $42(4)$ & $2(3)$ & $44(4)$ \\
\hline \multicolumn{4}{|l|}{ Procedure type } \\
\hline Nailbed repair & $75(7)$ & $3(4)$ & $78(7)$ \\
\hline $\begin{array}{l}\text { Soft tissue debride/closure/ } \\
\text { terminalisation }\end{array}$ & $322(32)$ & $17(24)$ & $339(31)$ \\
\hline Flexor repair & $79(8)$ & $14(19)$ & $93(8)$ \\
\hline Extensor repair & $66(6)$ & $6(8)$ & $72(6)$ \\
\hline$M U A \pm$ Cast/POP & $136(13)$ & $5(7)$ & $141(13)$ \\
\hline MUA+wire & $55(5)$ & $5(7)$ & $60(5)$ \\
\hline ORIF/ex fix & $165(16)$ & $11(15)$ & $176(16)$ \\
\hline Local/regional flap & $9(1)$ & $0(0)$ & $9(<1)$ \\
\hline Free flap & $0(0)$ & $1(1)$ & $1(<1)$ \\
\hline $\begin{array}{l}\text { Nerve decompression inc. } \\
\text { CTD }\end{array}$ & $3(<1)$ & $1(1)$ & $4(<1)$ \\
\hline Multiple flexors & $8(1)$ & $2(3)$ & $10(1)$ \\
\hline Multiple extensors & $6(1)$ & $1(1)$ & $7(1)$ \\
\hline Removal metalwork & $27(3)$ & $1(1)$ & $28(3)$ \\
\hline Vascular repair/replant & $6(6)$ & $3(4)$ & $9(1)$ \\
\hline Nerve repair \pm graft & $43(4)$ & $1(1)$ & $44(4)$ \\
\hline Other soft tissue & $8(1)$ & $1(1)$ & $9(1)$ \\
\hline Other bony & $2(<1)$ & $0(0)$ & $2(<1)$ \\
\hline Excision tumour/cancer & $11(1)$ & $0(0)$ & $11(1)$ \\
\hline \multicolumn{4}{|l|}{ Procedure site } \\
\hline Forearm & $93(9)$ & $10(14)$ & $103(9)$ \\
\hline Radius/Ulnar distal & $239(23)$ & $17(24)$ & $256(23)$ \\
\hline Carpus & $23(2)$ & $3(4)$ & $26(2)$ \\
\hline MCs including $\mathrm{CMCJs}$ & $145(14)$ & $8(11)$ & $153(14)$ \\
\hline P1 and P2 (zone 2 flexor) & $327(32)$ & $26(36)$ & $353(32)$ \\
\hline P3 (zone 1 flexor) & $194(19)$ & $8(11)$ & $202(18)$ \\
\hline \multicolumn{4}{|l|}{ Tourniquet } \\
\hline Yes, arm & $368(36)$ & $36(50)$ & $404(37)$ \\
\hline Yes, finger & $199(19)$ & $9(13)$ & $208(19)$ \\
\hline \multirow[t]{2}{*}{ No } & $427(42)$ & $23(32)$ & $450(41)$ \\
\hline & & & Continued \\
\hline
\end{tabular}




\begin{tabular}{|c|c|c|c|}
\hline \multicolumn{4}{|c|}{ Surgery-related characteristics n (\%)5 } \\
\hline \multirow[b]{2}{*}{ Variable } & \multicolumn{2}{|c|}{ Postsurgery complications } & \multirow[b]{2}{*}{ Total } \\
\hline & No $(n=1021)$ & Yes $(n=72)$ & \\
\hline Unclear & $27(3)$ & $4(5)$ & $31(3)$ \\
\hline \multicolumn{4}{|l|}{ Length of stay (days) } \\
\hline 0 & $873(86)$ & $42(58)$ & $915(84)$ \\
\hline 1 & $88(9)$ & $7(10)$ & $95(9)$ \\
\hline $2-10$ & $55(5)$ & $14(19)$ & $69(6)$ \\
\hline$>10$ & $4(<1)$ & $9(13)$ & $14(1)$ \\
\hline \multicolumn{4}{|l|}{ Follow-up } \\
\hline Face-to-face (surgical team) & $474(47)$ & $53(74)$ & $527(48)$ \\
\hline $\begin{array}{l}\text { Face-to-face (community } \\
\text { team) }\end{array}$ & $45(4)$ & $0(0)$ & $45(4)$ \\
\hline Face-to-face (therapy) & $155(15)$ & $11(15)$ & $166(15)$ \\
\hline Remote (surgical team) & $80(8)$ & $5(7)$ & $85(8)$ \\
\hline Remote (therapy) & $43(4)$ & $0(0)$ & $43(4)$ \\
\hline None & $222(22)$ & $3(4)$ & $225(21)$ \\
\hline
\end{tabular}

CTD, carpal tunnel decompression; ex fix, external fixation; MUA, manipulation under anaesthetic; ORIF, open reduction and internal fixation; $\mathrm{P} 1$, proximal phalanx; P2, middle phalanx; P3, distal phalanx; POP, plaster of Paris.

of the GEE model were very similar and therefore not shown).

\section{DISCUSSION}

The SARS-CoV-2 complication rate for upper limb surgery at the peak of the UK pandemic was low at $0.18 \%$ (two pneumonias). The overall 30 -day mortality was $0.09 \%$ (one SARS-CoV-2 pneumonia) and the 30-day mortality of day-case surgery was zero. Both these SARS-CoV-2-related complications occurred in patients who had been hospitalised for a prolonged

\begin{tabular}{lc}
\hline Table 6 Surgery outcomes & \\
\hline Variable & Total \\
\hline Status at 30 days & \\
\hline Alive and at home & $1086(99)$ \\
\hline Alive in hospital & $3(<1)$ \\
\hline Died (day 1-7) & $1(<1)$ \\
Reoperation (30 days) & $45(4)$ \\
\hline Complications (30 days) & $72(7)$ \\
\hline SARS-CoV-2-related pneumonia & $2(1)$ \\
\hline ICU admission & $1(<1)$ \\
\hline Sepsis & $1(<1)$ \\
\hline Superficial infection & $21(2)$ \\
\hline Deep infection & $12(1)$ \\
\hline Tendon rupture & $11(1)$ \\
\hline Nerve injury & $2(<1)$ \\
\hline Fracture displacement & $7(1)$ \\
\hline Chronic regional pain syndrome & $1(<1)$ \\
\hline Other & $15(1)$ \\
\hline *One individual had two complications-tendon rupture and a deep \\
infection. \\
ICU, intensive care unit.
\end{tabular}

Table 7 Logistic regression of factors associated with occurrence of 30-day complication

\begin{tabular}{llc}
\hline Variables & OR $(95 \% \mathrm{Cl})$ & P value \\
\hline Age & $1.02(1.00$ to 1.03$)$ & 0.007 \\
Gender & $0.86(0.50$ to 1.48$)$ & 0.595 \\
Comorbidity & $1.34(0.73$ to 2.43$)$ & 0.343 \\
Smoking & $1.56(0.80$ to 3.04$)$ & 0.188 \\
Urgency of surgery & $1.24(0.72$ to 2.12$)$ & 0.433 \\
Surgery indications & $3.71(1.91$ to 7.22$)$ & $<0.001$ \\
Tourniquet use & $0.86(0.48$ to 1.52$)$ & 0.596 \\
\hline Regional/General anaesthetic usage & $3.31(1.85$ to 5.93$)$ & $<0.001$ \\
\hline
\end{tabular}

period before their surgery, with the patient who died having been originally admitted for treatment of their SARS-CoV-2 pneumonia. At the peak of the pandemic, most centres (96\%) were screening patients for symptoms prior to admission but only $22 \%$ were carrying out SARS-CoV-2 swabbing, and within this context we found that upper limb surgery conferred a low risk to patients. These findings support not delaying any urgent surgery pending the results of SARS-CoV-2 testing, and they also support the continued resumption of elective upper limb practice, particularly when conducted without general anaesthesia.

The most common complications were bacterial surgical site infection in 33 cases (3\%), tendon rupture in 9 cases $(0.8 \%)$ and fracture displacement in 7 cases $(0.6 \%)$. These complication rates are comparable to reported rates in the literature. ${ }^{7-9}$ There was an association of greater age, surgical indication (infection) and regional or GA use with increased likelihood of a complication when analysed concurrently. Given infection was the most common complication, it is unsurprising that surgery for infection has a higher rate of postoperative complications, while the association with regional or GA is likely related to the more severe infections not being amenable to surgery under local anaesthetic only and the fact that more severe infections have a higher complication rate.

One of the limitations of this study is the relatively low proportion of patients within this cohort who were SARS-CoV-2 positive in the perioperative period. However, around $70 \%$ of community SARS-CoV-2 infections were asymptomatic in the $\mathrm{UK},{ }^{6}$ meaning that it is likely that a reasonable number of patients who underwent surgery in our cohort were SARS-CoV-2 positive but asymptomatic, given that SARS-CoV-2 prevalence was at its highest in April 2020. It is impossible to precisely estimate prevalence in the UK as random community testing was not carried out at this time point, and later seroprevalence studies are likely to significantly underestimate actual prevalence due to poor test sensitivity and the likelihood of other forms of non-antibody-related immunity. ${ }^{10}{ }^{11}$ The most recent high-quality UK data from the REACT study demonstrates a prevalence of around $0.2 \%$ in mid-May 
in similar age groups to those in our cohort, with a halving time of 8.6 days $^{6}$; assuming this halving time was fairly constant from late March to May then this would mean that prevalence in early April was around $5 \%-10 \%$. Therefore, it is reasonable to estimate that in the order of $3.5 \%-7 \%$ of the patients in our cohort were asymptomatic carriers of SARS-CoV-2. This is consistent with the recently published data relating to trauma patients from London, which demonstrated a rate of SARS-CoV-2 infection of $7 \%$ and a peak prevalence in early April 2020. ${ }^{12}$

The persistence of SARS-CoV-2 circulating in the population poses a challenge to patient safety, staff safety and efficient perioperative care. However, there is a strong rationale and evidence base to support not delaying urgent surgery for trauma and infection as this will increase the rate of surgical complications. ${ }^{7} 1314$ Many complex decisions involving trade-offs between the short-term risks from SARS-CoV-2 exposure, and long-term risks associated with delaying effective planned surgical interventions for other pathologies, with much difficulty arising from uncertainties relating to our knowledge of SARS-CoV-2. In addition, there is a need to prevent cross-infection between patients and staff when attending hospital. Given the high mortality that has been reported in patients undergoing major surgery in the presence of SARS-CoV-2 infection, pathways have been developed to minimise patients' risk of perioperative SARS-CoV-2 exposure. ${ }^{2}$ There are significant problems with the generalisability of these results to upper extremity surgery. There were very few cases of upper extremity surgery in COVIDSurg, and by only including SARS-CoV-2-positive patients (based on the same definition as used in our study), the study cannot provide an accurate estimate for absolute risk in all SARS-CoV-2-positive patients, as patients with asymptomatic infections would rarely have been included and emerging evidence demonstrates the asymptomatic group constitutes an overall majority of positive patients. ${ }^{15}$ We therefore argue that the findings of COVIDSurg should not be applied universally to patients undergoing upper extremity procedures due to the lesser physiological insult conferred by the procedure and, in the case of operations performed without GA, the absence of a simultaneous period of insult to the respiratory system conferred by anaesthesia.

The prevalence of SARS-CoV-2 in the UK in August 2020 was several levels of magnitude lower than in early April 2020. We would therefore argue that a more pragmatic approach is needed when it comes to the delivery of emergency and elective surgical services, and that this study can be of great use in terms of describing the risks within a pragmatic context. The context of our study is that in early April 2020 care pathways were not separate, patients were not advised against outdoor exercise and essential shopping trips and SARS-CoV-2 swabbing was not routinely performed. Within this context, the 30-day mortality was $0.09 \%$, and the incidence of SARS-CoV-2-related complications was low at $0.18 \%$, even in a cohort which consisted of many inpatient stays and large numbers of GA cases. Most of the surgery within our study was urgent and performed for trauma or infection. There is a strong argument that at the current time this urgent surgery should not be delayed solely for SARS-CoV-2 testing. The potential harms are clear in terms of delaying urgent surgery, particularly when it relates to contaminated wounds, tendon repair, infection and open fractures. ${ }^{71316}$

The separation of pathways has created many logistical difficulties in delivering both urgent and elective care, particularly in smaller centres which do not have separate sites to enable the physical separation patients having urgent and planned procedures. There are also many added costs due to additional processes such as the cost of swabbing, the logistics of administering the pathways and the costs of inefficiencies such as surgery postponed at the last minute due to a false positive swab test result. There are the additional social and economic impacts on the patients' families of requiring shielding of the whole household pending and following surgical treatments. ${ }^{3}$ Routine swabbing may have a false negative rate of around 30\%, meaning that at times it may falsely reassure, while as prevalence falls the proportion of positive tests that are true positives becomes increasingly minuscule. ${ }^{17}$ The data from this study support performing day case upper limb surgery not requiring GA without routine SARS-CoV-2 testing in healthcare settings in which patient groups can be kept physically separate. This would enable those patients being treated under local or regional anaesthetic to remain distant from other patients in whom the potential implications of contracting SARS-CoV-2 would be likely to be more serious, for example, this may be via the use of a dedicated minor operations facility.

We would therefore argue that, provided patients are screened for symptoms, kept isolated from other patients before and after surgery, wear a face mask in theatre and a basic level of PPE is worn by staff for all clinical encounters, day case upper limb surgery is safe. The findings will help clinicians inform patients regarding the relatively low real-world risks of undergoing upper extremity surgery while the threat of SARS-CoV-2 persists. The findings will also inform practice during future new surges, given that this study was carried out at the peak of the first wave of the pandemic.

\section{CONCLUSIONS}

The SARS-CoV-2-related complication rate for upper limb surgery at the peak of the first wave of the UK pandemic was low and the mortality was zero for patients admitted on the day of surgery. Urgent surgery should not be delayed pending the results of 
SARS-CoV-2 testing. Routine SARS-CoV-2 testing for day case upper limb surgery not requiring general anaesthesia may be excessive and have unintended negative impacts.

\section{Collaborators}

This study has been carried out collaboratively and should be published under group authorship. We ask that the study is published under the name 'The Corona Hands Collaborative' which contains:

Study group: Benjamin JF Dean ${ }^{\varphi}$, Nicholas Riley, Christopher Little, Maxim D Horwitz, Dan Armstrong, Chye Yew Ng, Michelle Spiteri, Jonathan $\operatorname{Cook}^{\phi \varphi}$ ( ${ }^{(}$denotes first author and ${ }^{\varphi \varphi}$ denotes senior author)

Writing group: Benjamin JF Dean, Nicholas Riley, Christopher Little, Maxim D Horwitz, Dan Armstrong, Chye Yew Ng, Michelle Spiteri, Emma Reay, Dominic Furniss, Andrew J Carr, Jonathan Cook

Statistical analysis: Jonathan Cook, Zachary Lim

The Corona Hands Collaborative: Michael Elvey, Colin McDonald (University College London Hospitals NHS Foundation Trust); Michael Elvey, Anna Panagiotidou, John Hardman (London Northwest Healthcare NHS Trust); James Duncan, Ian McNab, Mike Lamyman, Catriona Luney, Stephen Gwilym, Daisy Ryan, Alistair Reed (John Radcliffe Hospital and Nuffield Orthopaedic Centre, Oxford); Dan Armstrong (University Hospitals of Derby and Burton); Maxim D Horwitz, Robert Macfarlane (Chelsea and Westminster Hospital NHS Foundation Trust); Chye Yew Ng, Ahsan Akhtar (Wrightington, Wigan and Leigh NHS Foundation Trust); Ryan W Trickett, Kathryn Dayananda (Cardiff and Vale University Health Board); Ms Gillian L Eastwood, Ian McLaughlin Symon, Ms Antonia Hoyle (Stepping Hill Hospital Stockport); S H Bostock, Chris Baldwin, Raveen L Jayasuriya, Helen Richards, Megan E Birks (Sheffield Teaching Hospitals, Northern General Hospital); Claire Simpson, Matthew Brown (St John's Hospital, Livingston, West Lothian (NHS Lothian)); Simon M Johnson, Samuel Greensmith, Adam Couves (Ninewells Hospital Dundee and Perth Royal Infirmary, NHS Tayside); Alex J Nicholls, Zoe Lin (Hampshire Hospitals NHS Foundation Trust, Basingstoke); Dominic M Power, Bafiq Nizar (Birmingham Hand Centre, Queen Elizabeth Hospital Birmingham); Feiran Wu, Thomas Challoner (Heartlands, Good Hope and Solihull Hospitals, University Hospitals Birmingham); Tom Quick, Michael Fox, Kalpesh R Vaghela, Ashley Simpson, Rebecca Morgan (Royal National Orthopaedic Hospital, Stanmore); Suddhajit Sen (Noble's Hospital; Isle of Man); Adrian Murphy, Matthew Torkington, Taegyeong Tina Ha, Helen Wohlgemut (Glasgow Royal Infirmary); Christian West, Stephen Lipscombe, Taegyeong Tina Ha, Carole Chan, Rahul Bamal (St Helens and Knowsley NHS Trust, Whiston Hospital and St Helens Hospital); Claire Middleton, Shireen Ibish (Royal Berkshire Hospital, Reading); Jemma Rooker, Adam Smith (Great Western Hospital, Swindon); Clare J Wildin, Han Hong Chong, Mohamed Elbashir (University Hospital of Leicester NHS Trust); Clare Langley, Nicola Mackay (University Hospitals Coventry and Warwickshire); Duncan Avis, Rishi Das (Royal Hampshire County Hospital); Simon Robinson, Uthman Alao (Wirral University Teaching Hospital NHS Foundation Trust); Angus Maclean, Ullas Jayaraju (Royal Glamorgan Hospital, Llantrisant); Sue Fullilove, Juliana Hughes, Bruno Alcocer, Julia Sarginson (University Hospitals Plymouth); Oliver D Stone, Martin Shapev (Royal Devon and Exeter Hospital);
David J Murray, Albert Tang (Pennine Acute Trust, North Manchester and Fairfield Hospitals); Ladan Hajipour, Imad Madhi (Wythenshawe Hospital); Saurabh Odak, Amit Singh (University Hospital Morecambe Bay NHS Foundation Trust); Fizan Younis, William Fletcher (Royal Blackburn Hospital); Soham Gangopadhyay, Sathishkumar Balasubramanian (South Tyneside district Hospital and Sunderland Royal Hospital); Andrew Smith, YS Cho (East Kent University Hospitals Foundation Trust); Jeremy Rodrigues, Ramesh Chennagiri, Matthew Baldwin, Murray Forsyth (Buckinghamshire Healthcare NHS Trust);Karanjit Mangat, Alexander Denning (Warwick Hospital, South Warwickshire NHS Foundation Trust); Sunil Garg (James Paget University Hospital, Great Yarmouth); Nasser Hyder, Nastaran Sargazi (Leighton Hospital, Crewe); Andrew McKee, Jonathan W M Jones, Mike Dunne (North West Anglia Foundation Trust (Peterborough City Hospital, Stamford Hospital and Hinchingbrooke Hospital)); Simon Wimsey, Rishi Dhir (Princess Alexandra hospital, Harlow); Edwin P Jesudason, Ravishanker Tangirala (Ysbyty Gwynedd Penrhosgarnedd, Bangor); Rob Poulter, Zulfiqar Ali Haider (Royal Cornwall Hospital NHS Trust, Truro); Hannah Lennox-Warburton, Suresh Srinivasan, Amr El Bahi (Kettering General Hospital); James G Warner, Alastair J Konarski (Royal Bolton Hospital); Onur Berber, Yueyang Li (Whittington Healthcare NHS Trust); James Henderson, Alasdair Bott, Sameer Gujral (Southmead Hospital, North Bristol NHS Trust); Alex Cowey, Cezary Kocialkowski (Royal United Hospital, Bath); Sami Hassan, Amin Kheiran (Northampton General Hospital); Neeraj Ahuja, Attila Nemeth (Southport and Ormskirk Hospital NHS Trust); Andy Hacker, Alex Martin (Milton Keynes General Hospital); Adrian Chojnowski, Martha Ricketts (Norfolk and Norwich University NHS Trust); Patrick Goon, Madeline Warren, Martinique Vella Baldacchino, Clarence Yeoh (Lister Hospital, Stevenage); Shwan Henari (Horton General Hospital); Robert Turner, Rakan Kabariti (Princess Royal Hospital, Shrewsbury and Telford NHS Trust); Mohamed A Imam, Anshul Sobti (Rowley Bristow Orthopaedic Centre, Ashford and St Peter's NHS Trust); Claire Fitzgerald, Srinath Ranjit (Newham University Hospital, Barts Health NHS Trust); Noel Peter, Will Mason, Robyn Brown, Harry Akehurst (Cheltenham and Gloucester NHS Trust); Adeline Clement, Christopher Donoghue (Raigmore Hospital, Inverness (NHS Highland)); Aaron B Ng, Amy M Lindh (Pinderfields General Hospital); Grainne Bourke, Daniel J A Thornton, Joseph Stallard (Leeds General Infirmary); Ahid Abood, Nicholas G Rabey (Cambridge University Hospitals NHS Trust); David J Warwick, Michael J Newman (Royal South Hampshire Hospital, Southampton University Hospitals); David Lawrie, Alexandra EF Haddon (Aberdeen Royal Infirmary and Woodend Hospital, NHS Grampian); Edward Powell-Smith (Harrogate and District NHS Foundation Trust); Alexia Karantana, Sandeep Deshmukh (Nottingham University Hospitals NHS Trust); Harry Lewis, Serena Martin (Ulster Hospital, Northern Ireland); Sam Molyneux, Caitlin M Brennan (Royal Infirmary Edinburgh); Gavin Brigstocke, Joshua Luck (Frimley Park Hospital); Emma Reay, Christina Whitehead (James Cook University Hospital); Zakir Shariff, John Kiely (Bradford Teaching Hospitals NHS Foundation Trust); Matthew Torkington, Eoghan Donnelly (Queen Elizabeth University Hospital, Glasgow); Mohammed S Arshad, Pawan K Chajed (Royal Oldham Hospital); Gavan McAlinden, Rebecca Waterworth (South-Eastern HSC Trust); Ranjit Sehjal, Sheela Vinay (Maidstone and Tunbridge Wells NHS Trust).

Funding BJFD is part funded by the BMA's Doris Hillier grant. 
Competing interests None declared.

Patient consent for publication Not required.

Provenance and peer review Not commissioned; externally peer reviewed.

Data availability statement Data are available on reasonable request. Data are available on reasonable request to the lead author.

Supplemental material This content has been supplied by the author(s). It has not been vetted by BMJ Publishing Group Limited (BMJ) and may not have been peer-reviewed. Any opinions or recommendations discussed are solely those of the author(s) and are not endorsed by BMJ. BMJ disclaims all liability and responsibility arising from any reliance placed on the content. Where the content includes any translated material, BMJ does not warrant the accuracy and reliability of the translations (including but not limited to local regulations, clinical guidelines, terminology, drug names and drug dosages), and is not responsible for any error and/or omissions arising from translation and adaptation or otherwise.

Open access This is an open access article distributed in accordance with the Creative Commons Attribution Non Commercial (CC BY-NC 4.0) license, which permits others to distribute, remix, adapt, build upon this work noncommercially, and license their derivative works on different terms, provided the original work is properly cited, appropriate credit is given, any changes made indicated, and the use is noncommercial. See: http://creativecommons.org/licenses/by-nc/4. $0 /$.

\section{ORCID iD}

Benjamin John Floyd Dean http://orcid.org/0000-0002-89698868

\section{REFERENCES}

1 PHE. UK corona virus data. Gov website. Available: https:// coronavirus.data.gov.uk/

2 COVIDSurg Collaborative. Mortality and pulmonary complications in patients undergoing surgery with perioperative SARS-CoV-2 infection: an international cohort study. Lancet 2020;396:27-38-38.

3 BOA, 2020. Available: https://www.rcsed.ac.uk/media/681197/ boa-guidance-

4 NHS England. NHS England operating framework. Available: https://www.england.nhs.uk/coronavirus/wp-content/

5 NICE. COVID-19 rapid guideline: arranging planned care in hospitals and diagnostic services. NG179, 2020
6 STROBE. STROBE guidelines. Available: https://www.strobestatement.org

7 Reito A, Manninen M, Karjalainen T. The effect of delay to surgery on major complications after primary flexor tendon repair. J Hand Surg Asian Pac Vol 2019;24:161-8.

8 Minhas SV, Catalano LW. Comparison of Open and Closed Hand Fractures and the Effect of Urgent Operative Intervention. J Hand Surg Am 2019;44:65.e1-7.

9 Bannasch H, Heermann AK, Iblher N, et al. Ten years stable internal fixation of metacarpal and phalangeal hand fractures-risk factor and outcome analysis show no increase of complications in the treatment of open compared with closed fractures. J Trauma 2010;68:624-8.

10 Deeks JJ, Dinnes J, Takwoingi Y, et al. Antibody tests for identification of current and past infection with SARS-CoV-2. Cochrane Database Syst Rev 2020;6:CD013652.

11 Sekine T, Perez-Potti A, Rivera-Ballesteros O, et al. Robust T cell immunity in convalescent individuals with asymptomatic or mild COVID-19. Cell 2020.

12 Ajayi B, Trompeter A, Arnander M, et al. 40 days and 40 nights: clinical characteristics of major trauma and orthopaedic injury comparing the incubation and lockdown phases of COVID-19 infection. Bone Joint Open 2020;1:330-8.

13 Hull PD, Johnson SC, Stephen DJG, et al. Delayed debridement of severe open fractures is associated with a higher rate of deep infection. Bone Joint J 2014;96-B:379-84.

14 Symer MM, Abelson JS, Sedrakyan A, et al. Early operative management of complicated appendicitis is associated with improved surgical outcomes in adults. Am J Surg 2018;216:431-7.

15 Riley S, Ainslie KEC, Eales O, et al. Community prevalence of SARS-CoV-2 virus in England during May 2020: REACT study. medRxiv 2020.

16 Wu L, Chung KC, Waljee JF, et al. A national study of the impact of initial débridement timing on outcomes for patients with deep sternal wound infection. Plast Reconstr Surg 2016;137:414e-23.

17 Kader N, Clement ND, Patel VR, et al. The theoretical mortality risk of an asymptomatic patient with a negative SARS-CoV-2 test developing COVID-19 following elective orthopaedic surgery. Bone Joint J 2020;102-B:1256-60. 